\title{
RATE-OPTIMAL SCHEDULING OF RECURSIVE DSP ALGORITHMS BASED ON THE SCHEDULING-RANGE CHART*
}

\author{
Sonia M. Heemstra de Groot and Otto E. Herrmann \\ University of Twente, Faculty of Electrical Engineering \\ Laboratory for Network Theory \\ P.O.B. 217, 7500 AE Enschede, The Netherlands
}

\begin{abstract}
This paper presents a new method for rate-optimal scheduling of recursive DSP algorithms. The approach is based on the determination of the scheduling window of each operation and the construction of a "scheduling-range chart". The information in this chart is used during scheduling in order to optimize some quality criteria (number of hardware resources, latency, register life time) at the same time that a rate-optimal solution is guaranteed. An algorithm based on this approach is introduced. It can schedule cyclic as well as acyclic data flow graphs. The algorithm is powerful enough to solve optimally some problems for which other proposed methods fail.
\end{abstract}

\section{Introduction}

As a consequence of the complexity and speed of DSP algorithms, implementations that exploit parallelism are becoming more and more popular. This requires the support of suitable design tools. One of these should perform the scheduling and assignment of the operations to the hardware resources, which, for a big number of DSP algorithms, can be performed statically [5].

The algorithm description given as a signal flow graph can be mapped onto a data flow graph (DFG), where the nodes represent tasks or delay elements and the directed arcs represent the precedence relations. Associated to each task $T_{i}$ there is a processing time, $t c_{T_{i}}$. Figure 1 shows an example of a cyclic DFG. The DFG together with the set of hardware resources, that for reasons of simplicity will be considered as a set of identical processors, provide the model for the scheduling problem. In order to be able to exploit all the existing parallelism, the tasks will be assumed to be atomic. Communication delays are not considered.

When the main interest is speed, the scheduling goal is to min imize the sampling period. Without using pipelining, the minimum sampling period for execution of an iterative acyclic DFG is limited by the critical path, whenever enough computing power is available.

Similarly, for iterative cyclic DFG's there is a lower bound for the sampling period, imposed not by the critical path, but by the critical loop [10]. This bound is called iteration period bound and is denoted by $T_{0_{\min }}$. Schedules that can be executed at this sampling period are called rate-optimal schedules. Basically, three scheduling techniques that provide such schedules have been reported: maximum spanning tree $[10,11]$, search of cyclo-static schedules $[11,12]$, and optimum unfolding [7].

"This research has been partially financed by the Foundation FOM ("Innovative CAD Tools for IC Design", TEL 330408).

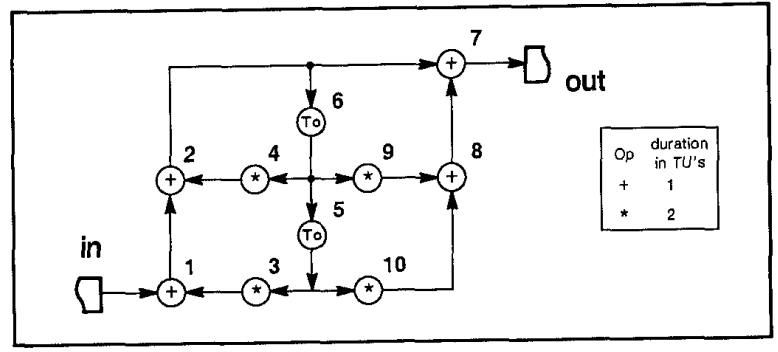

Figure 1: Cyclic data flow graph of a second order digital filter section. For reasons of simplicity, the duration of the operations is given in an abstract time unit, TU.

The first has the disadvantage that it does not try to optimize the number of processors, leading in general to solutions with suboptimal processor utilization [3]. The second consists of a depth first search of cyclo-static solutions, where the schedule is not only periodic in time but also in the processor space. In order to limit the search space, only solutions that are rate-optimal and requiring a number of processors equal to the processor bound, i.e. the minimum computing power necessary to execute the graph at optimal rate, are allowed. So, the solutions that this method provides are always processor optimal, when there exists a solution that can achieve such a bound. Otherwise, after exponential time with respect to the number of nodes, the algorithm finishes the search unsuccessfully. The third method consists of reducing the graph to an equivalent perfect-rate data-flow program that can always be scheduled rate-optimally. This is performed by unfolding with an optimal unfolding factor. The main disadvantage of unfolding is that the memory space necessary to store the program increases proportionally with the unfolding factor.

This paper presents an alternative approach for the rate-optimal scheduling of cyclic DFG's based on the determination of a scheduling range chart. The information in this chart is used during scheduling in order to optimize some quality criteria (number of hardware resources, latency, register life time) at the same time that a rate optimal solution is guaranteed.

\section{The Scheduling Range}

\subsection{Non-Iterative Acyclic DFG's}

For a non-iterative acyclic DFG (or iterative acyclic DFG without use of pipelining), an optimal schedule has the length of the critical path. Operations in the critical path cannot be deferred without affecting the length of the schedule. They don't have any mobility, and have to be scheduled in fixed time slots. Opera- 
tions that don't belong to the critical path can be scheduled in a range of time slots, or scheduling range. In an acyclic DFG, the scheduling range of an operation is finite and determined by its positions in the early and late schedule $[9,8]$. For an operation in the critical path, its early and late-schedule position are the same.

Based on information provided by the scheduling range, various scheduling algorithms for non-repetitive acyclic DFG's have been proposed. Examples are PERT [6], the scheduling strategies proposed by Ramamoorthy et. al. in [9], and the critical path algorithm for microcode compaction ${ }^{1}$ proposed by Ramamoorthy and Tsushita [4]. Another example is the recently proposed forcedirected scheduling [8] for behavioral synthesis of ASIC's that has been reported as very successful.

\subsection{Iterative DFG's}

In iterative DFG's it is possible to exploit not only the parallelism between operations of the same iteration, but also of different iterations. When the DFG is acyclic, pipelining can be used to reduce the sampling period $T_{0}$, which, for unlimited resources and neglecting the communication delays, becomes infinite ${ }^{2}$.

The speed bound for cyclic DFG's depends not only on the number and computational delay of the hardware resources, but also on the topology of the graph. The minimum sampling period $T_{0_{\min }}$ is determined by the critical loop [10] of the DFG.

\subsection{The Scheduling-Range Chart}

Given a reference operation whose position in the time schedule (assignment of the operation to specific, repetitive time-slot(s)) is fixed, it is possible to determine the relative allowed positions of the rest of the unscheduled operations. The set of positions where an operation can be scheduled is called the scheduling range. The scheduling-range chart displays this information for every operation in the DFG. The difference between the length of the scheduling range of an operation and its duration is called its mobility. The scheduling range of an operation of an iterative DFG can be finite or infinite. When a limit of the scheduling range has a welldetermined position, i.e. when all the predecessors or successors of the operation have been scheduled, it is called a fixed limit. The notation used in this paper is shown in Figure 2.

To build the scheduling-range chart, an operation is taken as a reference. This operation has no mobility. The scheduling range of the rest of the operations in the graph is determined by (the joint effects of) the following constraints:

- The precedence relation between operations. An operation cannot start its execution before all its predecessors have finished their execution. See Figure $3(\mathrm{a})$.

- Backward precedence relation between operations related by registers.

Operations which are in a common path, and separated by a node representing one or more delay elements should be scheduled such that the value stored in a register is not

\footnotetext{
${ }^{1}$ Do not confuse with the "critical path list scheduling" algorithm.

${ }^{2}$ The sampling period can be made shorter than the duration of the longest atomic operation by using direct blocking $[3,11]$, or cyclo-static techniques $[11,12]$.
}

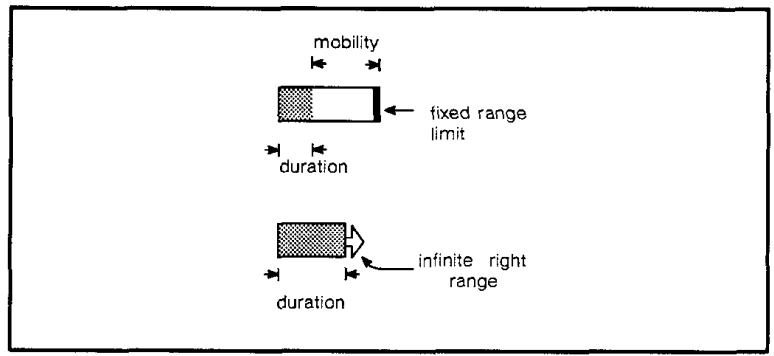

Figure 2: Notation for the scheduling range of operations.

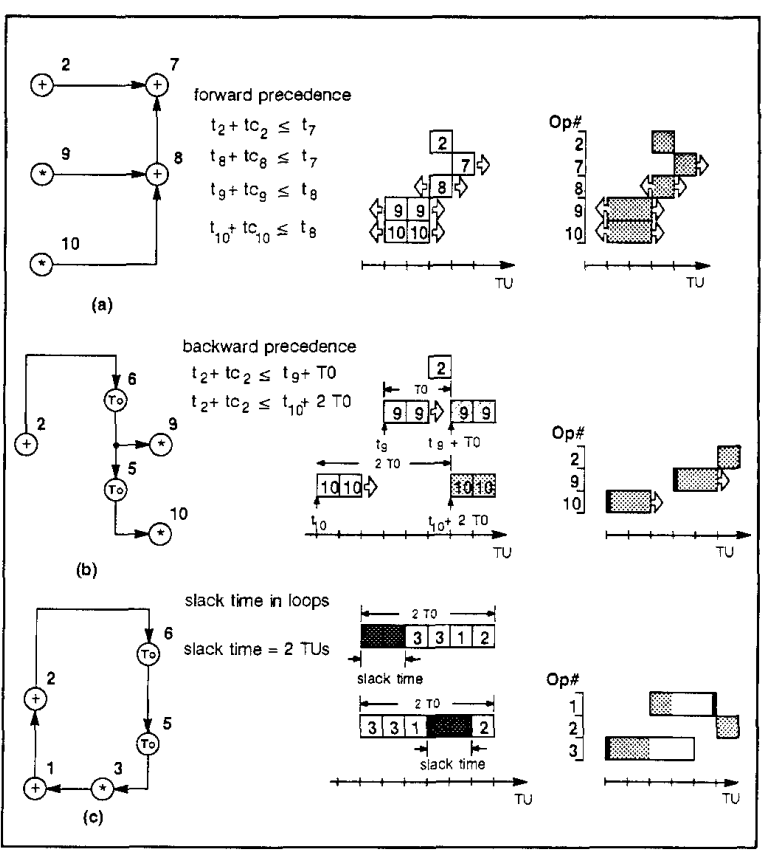

Figure 3: Scheduling range of operations for the DFG of Figure 1 . for $T_{0}=3 T U$ s, and operation 2 as reference. Forward precedence (a). backward precedence (b), and slack times in loops (c).

rewritten before the successor operation(s) has read it. Expressed in another way: let $T_{i}$ be an operation whose result is written to node $D_{l}$, a node representing a number $n$ of delay elements. Let $T_{j}$ be an operation that reads a value from $D_{l}$. Then, $T_{i}$ and $T_{j}$ should be scheduled such that the following relation is respected:

$$
t_{T_{i}}+t c_{T_{i}} \leq t_{T_{j}}+n T_{0}
$$

where

$t_{T_{k}}$ is the time when operation $T_{k}$ starts its execution.

$t c_{T_{k}}$ is the execution time of task $T_{k}$, and

$T_{0}$ is the iteration period.

This is shown in Figure $3(\mathrm{~b})$.

- Slack time in loops.

This is a consequence of the backward precedence relation among operations that belong to the same loop [2]. An operation in (a) loop(s) has a limited range mobility equal to the shortest slack time of the loops of which it is a member. The slack time of a loop $\mathcal{L}$ is defined by:

$$
\text { slack time }=n_{l} T_{0}-\sum_{T_{i} \in \mathcal{L}} t c_{T_{i}}
$$




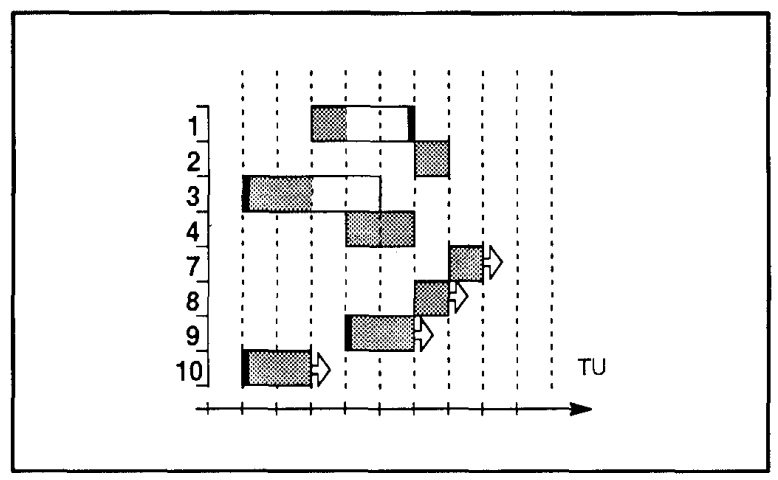

Figure 4: Scheduling range chart for the DFG of Figure 1, for $T_{0}=3 \mathrm{TUS}$, and operation 2 as reference.

where

$n_{l}$ is the number of delay elements in $\mathcal{L}$.

See Figure $3(\mathrm{c})$.

Figure 4 shows the scheduling-range chart corresponding to the DFG of Figure 1, for optimal sampling rate. Operation 2 has been taken as reference.

\section{Algorithm for the Minimization of Hardware Resources}

When both the sampling period and the number of resources are fixed, as is done in [12], a solution for the scheduling problem may not exist [2], as shown later with an example. In order to guarantee a solution either of the two parameters should be left free to be optimized by the algorithm. In [1] a scheduling algorithm is discussed that optimizes the sampling rate, for a fixed number of resources. Here an algorithm will be presented that minimizes Ue number of hardware resources, for a fixed sampling rate. The algorithm can be applyed to cyclic as well as to acyclic DFG's.

The scheduling algorithm is guided by the information displayed by the scheduling-range chart. In order to take into account the effect of the overlapping in time of the execution of operations of different iterations during the scheduling, the operations are grouped in $T_{0}$ equivalence classes [11], [3]. The operations in each equivalence class are executed in parallel.

The operations are scheduled by a priority given by their mobility in the scheduling-range chart. The $T_{0}$ equivalence 'classes are divided into levels; one processor per level is necessary. The algorithm assigns operations to equivalence classes and time slots by a priority determined by their scheduling range, and in such a way that the number of levels is optimized. Once this is performed for all the operations, the algorithm enters a new phase where the operations are assigned to processors.

$\Lambda$ short description of the algorithm is given below:

1. For acyclic DFG provide $T_{0}$.

For cyclic DFG determine the critical loop and $T_{0_{\text {min }}}$. Provide a sampling period such that $T_{0} \geq T_{0_{m i n}}\left(\right.$ make $T_{0}=$ $T_{0_{m i n}}$ for rate-optimal schedules).

2. Select a reference operation and determine the schedulingrange chart for $T_{0}$.

3. Partition the scheduling-range chart in sectors that corre- spond to each equivalence class.

4. Maintain for each equivalence class a pointer to the first available level; initially this pointer has a value 1 for all equivalence classes.

5. Select the unscheduleded operation with the shortest scheduling range. In case of equal length, give priority to an operation with a fixed-limit range. Select by label, if multiple operations have a range with fixed limit.

6. Assign the selected operation to the equivalence class(es) that has (have) the lowest first available level(s). Of course only those equivalence classes are considered, which are covered by the scheduling range of the operation. Since the level of a class is not associated to any processor, operations that cover more than one class can be assigned to different levels. Update the first available level of the affected equivalence classes.

7. Update the scheduling-range chart to incorporate the fixed position of the operation just scheduled.

8. Repeat steps 5,6 , and 7 until all operations have been scheduled.

9. Assign operations to processors.

(a) Sort operations according to their computational delay; the longest first; in case of equal duration sort by label.

(b) Maintain for each equivalence class a pointer to its first available level which now is associated to a processor. This pointer should be initialized to 1 for all equivalence classes.

(c) Remove the first operation from the list and assign it to the first processor level that has empty all the equivalence classes to which the operation has been assigned to. Update the first-available-level pointers.

(d) Repeat the last two steps until all the operations have been assigned to processors.

Figure 5 sequentially shows the previous steps for the rate-optimal scheduling of the DFG of Figure 1. Operation 2, that belongs to the critical loop, is used as reference. $T_{0}=T_{0_{\text {min }}}=3$ TU's, so there are three equivalence classes: 0,1 , and 2 , which are assigned sequentially to sectors in the chart. After this step, operations 2 and 4, that don't have mobility have been automatically assigned to classes 0 , and 1 and 2 respectively, in level 1 (Figure $5(\mathrm{a})$ ). The next selected operation is 1 . There are no more empty levels, so a new level is created. The operation is scheduled giving preference to the position where the fixed limit is, filling level 2 of class 2 ; the chart is updated (Figure $5(\mathrm{~b})$ ). The algorithm continues with 3 . Of the three possible positions, (1-2), (2-0), and (0-1), the algorithm chooses the first in order not to create a new level (Figure 5(c)). The algorithm continues in this way until all the operations have been assigned to relative time slots (time schedule), and to equivalence classes (Figure $5(\mathrm{~g})$ ). The result of the processor assignment phase is shown in Figure $5(\mathrm{~h})$; the processor utilization is $100 \%$. Figure 5(i) visualizes the overlapping in the execution of three consecutive iterations.

Figure 6 shows the rate-optimal schedule for the same DFG example, obtained by using the maximum spanning tree method $[10$, 11 , which requires 5 processors.

Figure 7(a) shows an example of a cyclic DFG which does not have a rate-optimal solution for a number of processors equal to the processor bound $[11,12], P_{0}=2$. Inspection of the schedulingrange chart (Figure $7(\mathrm{~b})$ ), shows that the only operation that has mobility is 7 . It can only be assigned to classes (1-2), or (2$3)$. Both possible schedules require 3 processors. Therefore, the search method proposed in $[11,12]$ fails in this case. 


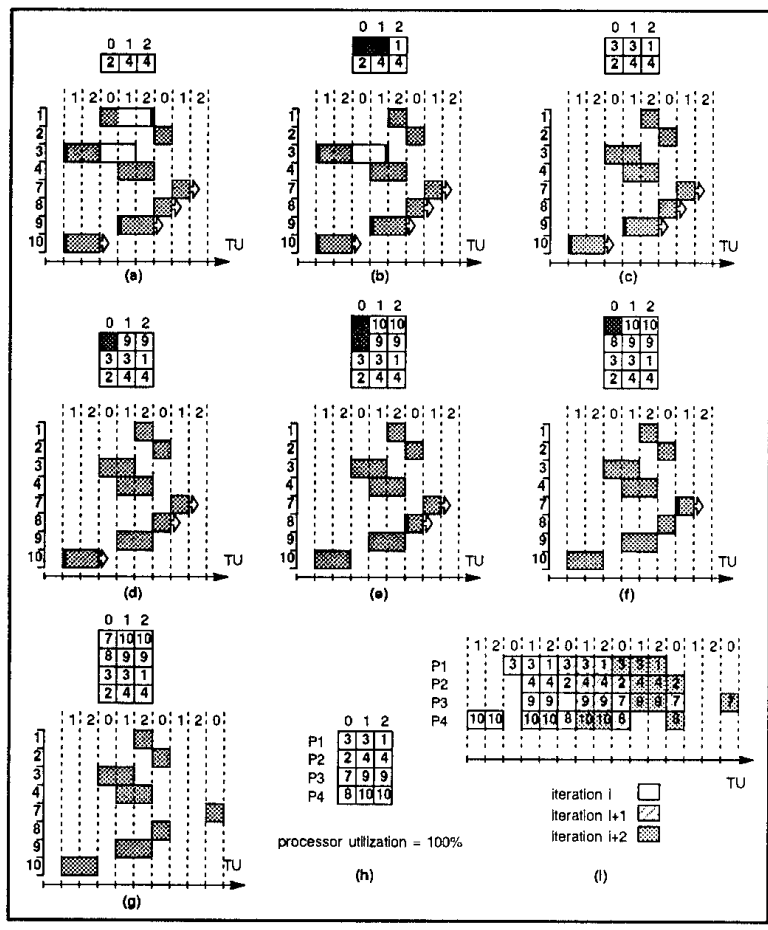

Figure 5: Scheduling algorithm. Sequential steps of the timescheduling and equivalence-class assignment, (a) to ( $g$ ). Processor assignment $(h)$. Processor execution of three consecutive iterations (i).

\section{Conclusions}

A new approach for rate-optimal scheduling of recursive DSP algorithms has been introduced. It is based on using the information of the scheduling range of the operations, and is an intent to overcome the limitations of other proposed methods. Operations can be moved within their scheduling range and scheduled such that the hardware resources are optimized. An algorithm based on this approach as been proposed. The algorithm, although simple, is powerful enough to solve many problems optimally.

Even more sophisticated methods, that schedule considering the effect of the scheduling of an operation on the total schedule can be applied, by converting the scheduling-range chart into a scheduling-range distribution chart where the height of the rectangle that determines the range is determined by the probability that the operation will be scheduled in that time slot. The area should be equal to the duration of the operation. In practice, the life time of the variables should be minimized. So, the scheduling range is finite, an a modification of the force-directed method [8] can be applied.

\section{References}

[1] S.M. Heemstra de Groot. Maximum Throughput Scheduling with Limited Resources for Iterative Data Flow Graphs by Means of the Scheduling-Range Chart. Technical Report, University of Twente, November 1989. Internal Report EL-BSC-89N196.

[2] S.M. Heemstra de Groot. Scheduling of Iterative Data Flow Graphs by Means of the Scheduling-Range Chart. Technical Report, University of Twente, October 1989. Internal Report ELBSC-89N181.

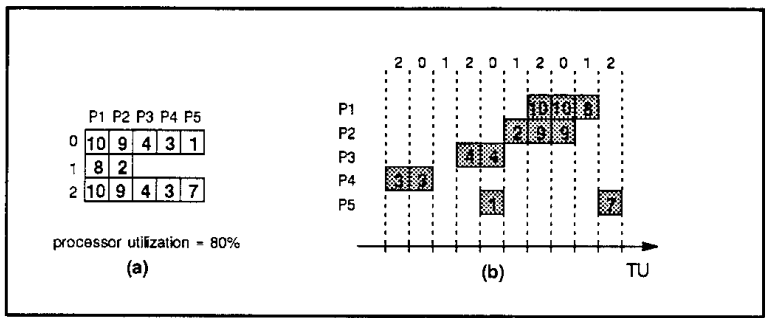

Figure 6: Maximum-spanning-tree rate-optimal scheduling. Assignment of operations to equivalence classes and processors (a), and schedule (b).

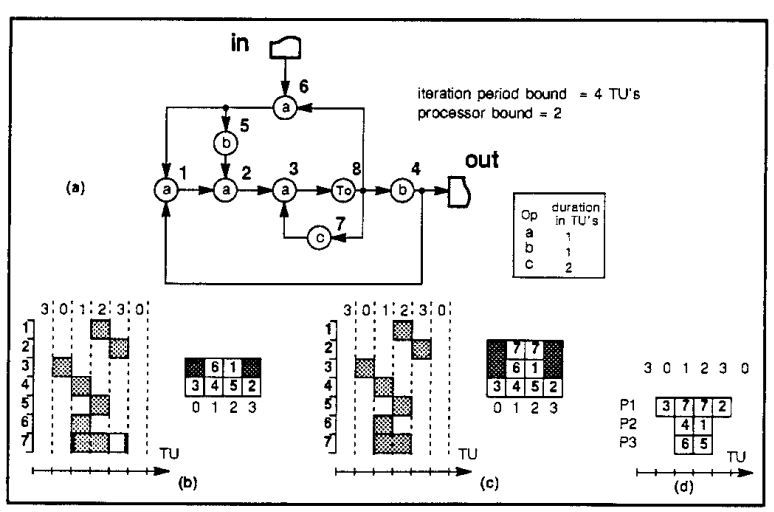

Figure 7: Cyclic DFG (a), corresponding scheduling-range chart (b), equivalence classes and processor assignment (c), and rate-optimal schedule (d).

[3] S.M. Heemstra de Groot and O.E. Herrmann. Evaluation of some multiprocessor scheduling techniques of atomic operations for recursive DSP graphs. In Proceedings of the European Conference on Circuit Theory and Design, pages 400-404, September 1989.

[4] D. Landskov, S. Davidson, and B. Shriver. Local microcode compaction techniques. ACM Computing Surveys, 12(3):261-294, September 1980 .

[5] E.A. Lee and D.G. Messerschmitt. Static scheduling of synchronous data flow programs for digital signal processing. IEEE Transactions of Computers, C-36(1):24-35, January 1987.

[6] R.I. Levin and Ch. A. Kirkpatrick. Planning and Control with PERT/CPM. McGraw-Hill, 1966.

[7] K.K. Parhi and D.G. Messerschmitt. Rate-optimal fully-static multiprocessor scheduling of data-flow signal processing programs. In Proceedings of the 1989 IEEE International Symposium on Circuits and Systems, pages 1923-1928, May 1989.

[8] P.G. Paulin and J.P. Knight. Force-directed scheduling for the behavioral synthesis of ASIC's. IEEE Transactions on ComputerAided Design, 8(6):661-679, June 1989.

[9] C.V. Ramamoorthy, K.M. Chandy, and M.J. Gonzalez. Optimal scheduling strategies in a multiprocessor system. IEEE Transactions on Computers, C-21(2):137-146, February 1972.

[10] M. Renfors and Y. Neuvo. The maximum sampling rate of digital filters under speed constraints. IEEE Transactions on Circuits and Systems, CAS-28(3):196-202, March 1981.

[11] D.A. Schwartz. Synchronous Multiprocessor Realizations of Shift Invariant Flow Graphs. PhD thesis, Georgia Institute of Technology, 1985

[12] D.A. Schwartz and T.P. Barnwell. Cyclo-static multiprocessor scheduling on the optimal realization on shift-invariant flow graphs. In IEEE International Conference on Acoustics, Speech and Signal Processing, pages 1384 - 1387, 1985. 